\title{
New Approach to Quantifying Developmental Variation in the Dentition Using Serial Microtomographic Imaging
}

\author{
GAL AVISHAI, ${ }^{1}$ RALPH MÜLLER, ${ }^{2}$ YANKEL GABET,${ }^{3}$ ITAI BAB,${ }^{3}$ URI ZILBERMAN, ${ }^{1}$ AND PATRICIA SMITH ${ }^{1 *}$ \\ ${ }^{1}$ Laboratory of Bio-Anthropology \& Ancient-DNA, Institute of Dental Sciences, Faculty of Dental Medicine, \\ Hebrew University, Jerusalem, Israel 91120 \\ ${ }^{2}$ Institute for Biomedical Engineering, Swiss Federal Institute of Technology (ETH) and University of Zürich, \\ CH-8044, Zürich, Switzerland \\ ${ }^{3}$ Bone Laboratory, Institute of Dental Sciences, Faculty of Dental Medicine, Hebrew University, Jerusalem, Israel 91120
}

KEY WORDS serial microtomographic imaging; tooth development; deciduous molar

\begin{abstract}
Dental morphogenesis and cellular differentiation are expressed in the fully formed tooth by the topography of the dentin-enamel junction and outer enamel surface. These boundaries can be differentiated using a variety of imaging systems. In this study, we used serial microCT imaging to provide accurate 3D reconstructions of developing lower human second deciduous molars. These were used to quantify the volume of enamel and dentin of individual cusps in relation to basal crown height. As growth and differentiation proceed apically, the spatial orientation of cusp tips and their bases were used to estimate their order of initiation and coalescence. We found that the order of coalescence differed from the order of initiation. We also found that dentin cusp height and volume as well as rate and quantity of enamel apposition varied along mesio-distal and bucco-lingual axes, and were independent of order of initiation and duration of growth. These results demonstrate that the potential for variation in crown size and form is maintained throughout development. We propose that the microCT model developed in this study constitutes a new approach for the investigation of developmental variation and its contribution to phylogenetic variation expressed in crown form and size. Microsc. Res. Tech. 65:263-269, $2004 . \quad$ ๑ 2005 Wiley-Liss, Inc.
\end{abstract}

\section{INTRODUCTION}

Molecular mechanisms are being increasingly invoked to interpret phenotypic diversity expressed in crown form and size of mammalian molar teeth, using the traditional trigon (id)-talon (id) partitioning of early and late stages of growth. However, the pattern of tooth formation is such that finer discrimination between developmental stages can be recognized in the fully formed tooth using imaging techniques (Conroy and Vannier, 1991; Smith et al., 1997, 1998; Zilberman et al., 2004), histological sections (Macho and Berner, 1993), or differential decalcification of either enamel or dentin (Korenhof, 1979; Kraus, 1952; Sasaki and Kanazawa, 1998). These methods distinguish between growth attributable to cell division on the one hand and the further modification of crown size and form due to enamel matrix laid down by the ameloblasts on the other. However, they do not provide a means of assessing growth rates within the tooth. All hominid lower permanent molars represent variations on the fivecusped Dryopithecine pattern first described by Gregory (1922). Variation in crown form and size are associated with differences in the volume of the combined dentin-pulp complex, relative proportions of individual cusps, definition of ridges connecting them, groove pattern and enamel thickness (Korenhof, 1979; Kraus, 1952; Sasaki and Kanazawa, 1998), but are not necessarily correlated with tooth size or enamel thickness (Beynon, 1992; Beynon and Dean, 1987; Beynon and Wood, 1987; Faerman et al., 1994; Smith et al., 2003).

Tooth germs are initiated as localized swellings of the dental lamina. They are rapidly surrounded by mesenchyme of neural crest origin (Lumsden, 1988), leading to differentiation of the enamel organ and dental papilla. These processes are complex and involve numerous growth factors, regulating epithelial-mesenchymal interactions that are shared with other organs (Thesleff, 2003). The appearance of each of the developing cusps in the molars is associated with a nonproliferating group of cells called the enamel knot, which controls many of the events associated with cuspal development. The knots appear when a certain molecular threshold is reached, namely, an upregulation of p21, which is signaled by BMP-4 (Jernvall and Jung, 2000). Mitosis around the enamel knot causes the internal enamel epithelium around it to grow apically in a drape-like fashion, forming a soft tissue cusp. The signal for mitosis is provided by growth factors from the enamel knot, mainly fgf-4 (Jernvall et al., 1998). Signaling for mitosis ceases when the enamel knot cells undergo apoptosis and cells of the inner enamel epithelium and adjacent dental papilla differentiate into matrix-secreting ameloblasts and odontoblasts that produce the calcified matrix (enamel and dentin, respectively). This process progresses apically as addi-

*Correspondence to: Patricia Smith, Laboratory of Bio-Anthropology \& Ancient-DNA, Institute of Dental Sciences, Faculty of Dental Medicine, Hebrew University-Hadassah, Jerusalem, Israel, POB 12272, 91120.

E-mail: pat@cc.huji.ac.il

Contract grant sponsor: Israeli Science Foundation; Contract grant number: 032-5302.

Received 16 September 2004; accepted in revised form 25 October 2004

DOI 10.1002/jemt.20131

Published online in Wiley InterScience (www.interscience.wiley.com). 
tional cells undergo differentiation along the calcifying front.

The final position and size of each cusp, defined by the dentin-enamel junction (DEJ), reflects the summation of mitosis prior to coalescence between adjacent cusps by advancing waves of calcification. Since the entire tooth is growing apically, the height of the DEJ at the coalescence of adjacent cusps reflects their order of union. Continued basal growth of the tooth germ increases the volume of the dentin-pulp complex, while continued enamel apposition on the surface of the crown further increases the total volume of the tooth.

The difference in radio-opacity of enamel and dentin means that the boundary between them can be accurately visualized by different imaging systems, including serial computerized tomography. Smith et al. (1997, 1998) used this approach to compare growth expressed at the DEJ with that on the outer surface of the crown. The accuracy of this approach, however, was limited by the thickness of the slices obtained $(1.2 \mathrm{~mm})$. Desktop microcomputed tomography (microCT) overcomes this problem. It provides excellent resolution, on the order of that obtained using standard histological techniques, but has the advantage of being a noninvasive procedure. The microCT model $\mu$ CT40 (Scanco Medical, Bassersdorf, Switzerland) is an accurate, noninvasive system giving results similar to those obtained using standard histological techniques (Muller et al., 1996, 1998). Recent applications include research on osteoporosis (Ruegsegger et al., 1996), the effect of parathyroid hormone on bone generation in ovariectomized mice (Alexander, 2001), 3D modeling of root canal anatomy in endodontics (Peters et al., 2000), and quantification of periradicular bone loss surrounding infected teeth (von Stechow et al., 2003; Gabet et al., 2004; Muller et al., 1996, 1998; Peters et al., 2000; Ruegsegger et al., 1996; von Stechow et al., 2003).

In this study we used high-resolution serial microCT sections to differentiate between enamel and dentin in order to model growth and enamel matrix apposition in the second lower deciduous molar. The serial microCT scans were used to construct 3D images of a crosssectional sample of developing tooth germs and to quantify individual cusps and basal crown volume of enamel and dentin. The underlying hypothesis of this study is that growth attributable to cell division is expressed by the volume of the tooth enclosed by the DEJ, while ameloblast function is reflected in the volume of the enamel. Since cusp initiation and development of the basal portion of the crown are highly ordered, quantification of these parameters in individual cusps and in the basal crown enables us to apportion variation in successive stages of tooth development. This constitutes a novel approach to the investigation of the link between developmental processes and phenotypic variation.

\section{MATERIALS AND METHODS}

The sample used in this study was based on tooth germs excavated from different archeological sites in Israel. The stage of development of the tooth germs used ranged from minimal (three cusps present) to crown completion. This cross section of different developmental stages allows for deduction of growth processes at different stages and at different locations within the tooth. They were chosen to represent different stages of dental development with crown height ranging from $2.4 \mathrm{~mm}$ (three cusps united, estimated age 7.5 lunar months) to $6.5 \mathrm{~mm}$ (crown complete, estimated age 10 months and older). Age estimations for successive stages of development were based on Butler (1998), Kraus and Jordan (1965), and Liversidge (1993).

Criteria for inclusion in the sample group were: excellent preservation, no visible cracks or breakage, and no attrition. No discrimination between right and left teeth was made (14 specimens were right and 10 were left). All teeth were from different individuals; right and left teeth from the same individuals were not used. The tooth germs and teeth were cleaned using a soft brush and were not treated in any other way.

\section{Scanning}

Specimens were scanned in a MicroCT model $\mu$ CT40 (Scanco Medical). This apparatus contains a microfocus X-ray tube (with a focal spot of $10 \mu \mathrm{m}$ ), a turntable system for mounting and rotating the specimens, and a linear array CCD detector connected to a computer. The teeth were placed in a specialized holding tube of the microCT so that the tips of the three mesial cusps (protoconid, metaconid, and hypoconid) were in the same plane and a preview scan was performed to provide a low-resolution image of the tooth to define the upper and lower border of each scan.

Specimens were then scanned at $16 \mu \mathrm{m}$ resolution with the energy set to $60 \mathrm{keV}$. Average scan time for each tooth was 3 hours. Values used to differentiate between dentin and enamel were 500 for enamel and 400 for dentin, on a radio-opacity scale of $1-1,000$. The value chosen to define the outer surface of the enamel was 30 to allow for annulment of dust particles and other residues on the specimens. Standardization of values has been shown to give extremely accurate discrimination of tissues with different degrees of mineralization (Muller et al., 1996, 1998).

Possible errors in locating landmarks were assessed by repeating 20 measurements on 18 teeth, with a 6 -week interval between measurements. No significant differences were found. Error of measurement was found to be $0.9 \%$. The accuracy of contouring was assessed digitally contouring one tooth, comprising 300 slices, twice in order to estimate the error of measurement. Error of measurement was found to be $1.19 \%$.

The end result of the scan was a series of slices, each one $16 \mu$ thick. The first slice was at the apical end of the crown or tooth germ and the last slice was at the tip of the highest cusp. This series of scans comprised the "Z" axis of the analysis. Each of the slices along the Z-axis showed the configuration of the tooth germ at a specific height (Fig. 1). The visual image of each slice was compiled of an $\mathrm{X}, \mathrm{Y}$ grid at $16 \mu$ resolution. The 3D $X, Y, Z$ grid was used to create an accurate 3D model in which each cusp was defined three dimensionally, with a specific tip and a base defined by a line joining the points of union with two adjacent cusps (Fig. 2). Since the points of union of each cusp to neighboring cusps are at different heights, this line is an estimated projection of the cusp base. 


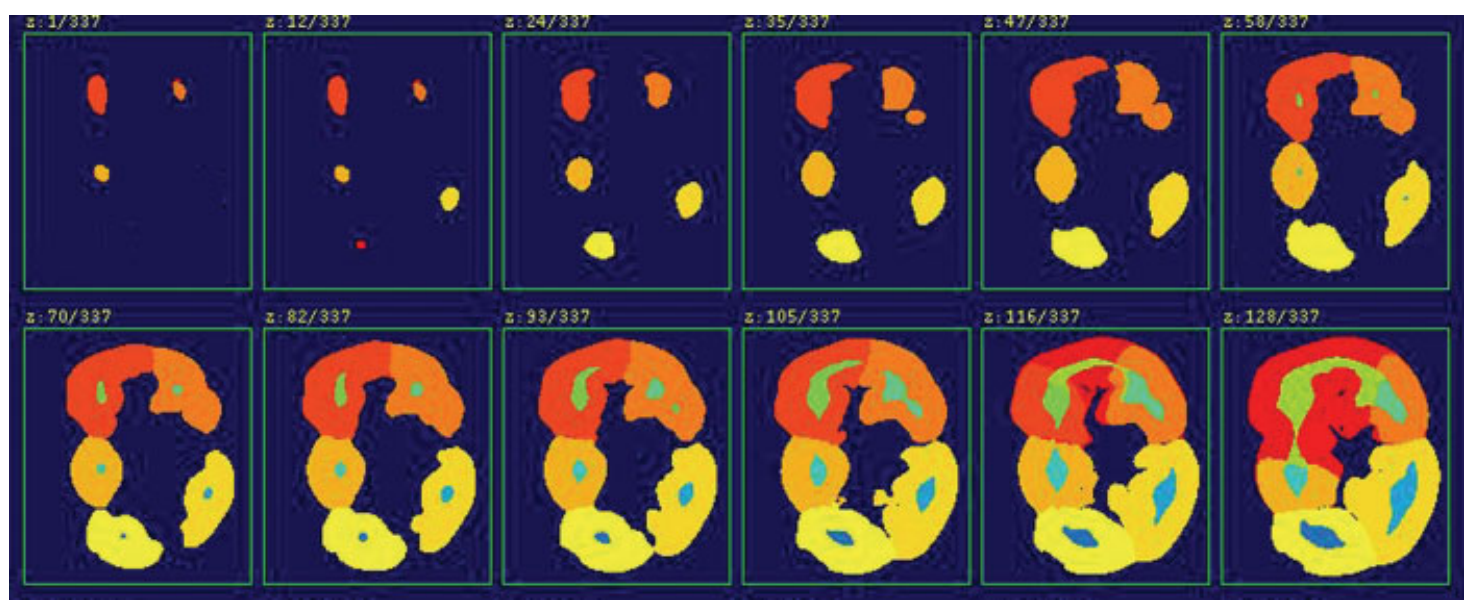

Fig. 1. Selected scans showing enamel and dentin at different locations along the Z-axis. Note pattern of coalescence between cusps. The protoconid is in the top left-hand corner. The cusps are color-coded (red, protoconid; yellow, metaconid; green, hypoconid; dark blue, entoconid; brown, hypoconulid).

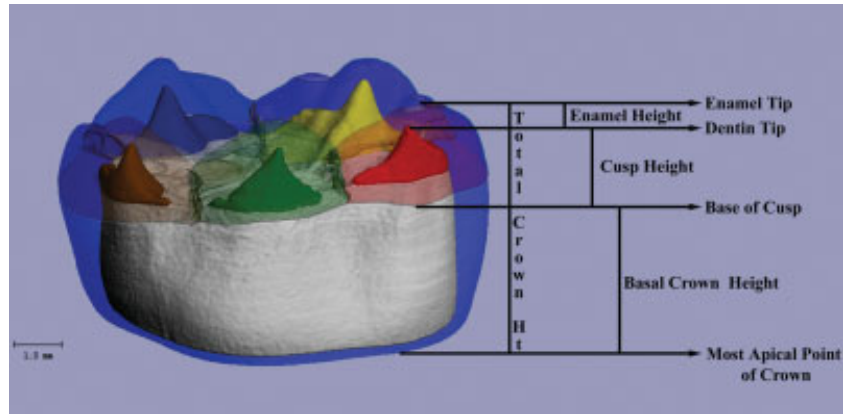

Fig. 2. Three-dimensional reconstruction of a deciduous lower second molar created from microCT serial sections. The reconstruction shows the spatial location of points defined and differentiation of enamel (translucent) and dentin (solid color). Measurements taken for each cusp were enamel height (EH), cusp height (DCH), basal crown height $(\mathrm{BCH})$, and total crown height (TCH). The cusps are colorcoded (red, protoconid; yellow, metaconid; green, hypoconid; dark blue, entoconid; brown, hypoconulid)

The model uses the topography of the DEJ to identify three phases of growth contributing to crown size and form defined as:

- Dentin Cusp Height (DCH): height of each cusp from tip to base measured along the DEJ, where the base is defined as the average height of coalescence of adjoining cusps.

- Enamel Height (EH): height of enamel from the dentin tip to the enamel tip.

- Basal Crown Height (BCH): basal portion of the crown, from the base of the cusp to the base of the crown.

Statistical analyses were carried out using Microsoft Excel and SPSS 12.0. Comparisons of dentin and enamel cusp height and volume and basal crown height within teeth were carried out using the Mann-Whitney nonparametric test. Least mean squares analyses were used to analyze the association between crown height and other parameters studied.

\section{RESULTS}

Dentin cusp height (DCH) was significantly greater in the lingual cusps than in the buccal cusps $(P<0.05)$, with the entoconid tallest and the protoconid shortest (Table 1). Thus, bucco-lingual orientation of cusps and not their order of initiation determined DCH. There was no association between dentin cusp height and stage of crown formation, expressed as crown height. Least mean squares analyses gave values of $R^{2} \leq$ 0.1. This was expected, since DCH defined from cusp tip to coalescence was complete in all specimens studied.

Enamel height over the cusp tips (EH) and enamel volume (EV) in all cusps were correlated with crown height (Fig. 3a,b). In the youngest tooth germs studied here (approximate age 7.5 months in utero), $\mathrm{EH}$ over cusp tips was greatest in the protoconid, but the further rate of increase of $\mathrm{EH}$ in this cusp was low relative to that observed in other cusps. By 6 months of age (crown height $\sim 5 \mathrm{~mm}$ ) $\mathrm{EH}$ in the distal cusps was greater than that of the mesial cusps and in teeth with completed crowns (crown height greater than $6 \mathrm{~mm}$ ) $\mathrm{EH}$ was thickest in the distal cusps. Values for enamel volume showed a slightly different pattern, since it reflected the overall shape of the cusp and specifically basal area. In the completed teeth EV was greatest in the entoconid and least in the hypoconid and hypoconulid (Figs. 3b, 4).

Basal crown height in teeth with crowns completed was inversely proportional to dentin cusp height. Mean differences in height between the base of cusps at coalescence, using the protoconid-hypoconid coalescence point as 0 , were as follows: $0.19 \mathrm{~mm}$ between metaconid and hypoconid; $0.18 \mathrm{~mm}$ between hypoconid and hypoconulid; $0.49 \mathrm{~mm}$ between hypoconulid and entoconid; and $0.78 \mathrm{~mm}$ between metaconid and entoconid. As can be seen in Table 1, the relative height of the DEJ at coalescence found here 
TABLE 1. Timing of cusp initiation, calcification, and union in relation to dentin cusp height (weeks in utero)

\begin{tabular}{|c|c|c|c|c|c|c|c|c|c|}
\hline Cusp & $n$ & $\begin{array}{c}A \\
\text { Cusp } \\
\text { initiation }\end{array}$ & $\begin{array}{c}B \\
\text { Initiation of } \\
\text { calcification }\end{array}$ & $\begin{array}{c}C \\
1^{\text {st }} \\
\text { Union }\end{array}$ & $\begin{array}{c}D \\
2^{\text {nd }} \\
\text { Union }\end{array}$ & $\begin{array}{c}(A \text { to } B) \\
\text { Soft tissue } \\
\text { development }\end{array}$ & $\begin{array}{l}(B \text { to } D) \\
\text { Average duration } \\
\text { of calcification }\end{array}$ & $\begin{array}{c}(A \text { to } D) \\
\text { Total time of } \\
\text { development }\end{array}$ & $\begin{array}{l}\text { Mean dentin } \\
\text { cusp height } \\
(\mu \mathrm{m})\end{array}$ \\
\hline Protoconid & 24 & 14 & $18-22$ & 30 & 32 & 6 & 12 & 18 & $1,568 \pm 156$ \\
\hline Metaconid & 24 & 15 & 23 & 30 & 32 & 8 & 9 & 17 & $1,990 \pm 373$ \\
\hline Hypoconid & 24 & 15 & 26 & 30 & 32 & 11 & 6 & 17 & $1,649 \pm 140$ \\
\hline Entoconid & 21 & 16 & 28 & $32-36$ & $32-36$ & 12 & 6 & 18 & $2,163 \pm 255$ \\
\hline Hypoconulid & 21 & 17 & 28 & 32 & $32-36$ & 11 & 6 & 17 & $1,798 \pm 214$ \\
\hline
\end{tabular}

Note that mean dentin cusp height is independent of time of initiation or duration of development. Soft tissue development in the distal, later-appearing cusps continues longer than in the protoconid and metaconid, but the duration of calcification from initiation to coalescence is shorter.

follows the sequence described by Kraus and Jordan (1965).

\section{DISCUSSION}

Previous studies of phenotypic variation in dentition have focused on crown form and used cusp location and height to differentiate between early and late stages of development (Hlusko et al., 2004; Jernvall et al., 1998; Jernvall, 2000; Jernvall and Jung, 2000; Polly, 1998). The model developed here enables us to partition growth more precisely and to identify successive stages of development from examination of fully formed teeth. As demonstrated in Table 1, the timing of cusp initiation is not correlated with final cusp height. The duration of soft tissue proliferation relative to calcification increases with order of appearance of the respective cusps. The protoconid and hypoconid coalesce at the same time or even earlier than the protoconid and metaconid, and there is a 2 -week delay between union of these three cusps and the two distal cusps. Our data show that the relative height of cusp tips is staggered according to their order of initiation and that the relative height of the bases of the cusps reflects their order of coalescence described by Kraus and Jordan (1965). However, our data also show that neither DCH, EH, nor cusp area are correlated with the order of initiation or duration of growth of individual cusps.

The metaconid and hypoconid cusps are initiated at approximately the same time, but the metaconid is significantly taller than the hypoconid or protoconid, although calcification in the metaconid begins some 3 weeks earlier than in the hypoconid (Table 1). Soft tissue proliferation in the talonid cusps continues for almost twice as long as in the protoconid and metaconid, but the talonid cusps calcify more rapidly from tip to base. There is only a 1 -week difference between the hypoconulid and entoconid in initiation of the soft tissue cusps, and both begin calcifying at the same time. However, they are significantly different in height and volume when completed.

Dentin cusp height was significantly shorter in the buccal cusps than in the lingual cusps, and the reverse applies to basal crown height. No significant differences were found between height of the protoconid, hypoconid, and hypoconulid cusps in dentin cusp height, but in the completed crown enamel height in both distal cusps was thicker than on the mesial cusps. As shown in Figure 3, this appears to result from faster, more prolonged enamel apposition in the distal cusps. These results show that there is a shape gradient along the bucco-lingual axis and a size gradient along the mesio-distal axis. Both are associated with differences in dentin cusp height and shape and in enamel volume and enamel height over cusp tips.

The model presented here assumes that dentin cusp height is related to growth of individual cusps, which can only occur until coalescence. Since growth proceeds apically, differences in the timing of coalescence are associated with increased basal crown area and height differences at the DEJ reflected in fissure depth. The three mesial cusps (protoconid, metaconid, and hypoconid) unite by the 30th gestational week, but only coalesce with the distal cusps (hypoconulid and entoconid) some 2 weeks later. During this period the entire tooth is growing apically, with the three mesial cusps growing as one unit relative to the two distal cusps. Thus, the hypoconid is incorporated into a modified trigonid, while the other two-talonid cusps grow independently. The result is expressed in the angulation of the mesial compared to the distal cusps, the distance between cusp tips, and the relative height of the base of cusps at coalescence. Localized differences in basal crown growth, after coalescence of all cusps, will tilt the occlusal surface of the entire tooth. This is further modified by addition of crown height and volume by enamel apposition.

The discrepancy between the order of initiation and resultant dentin cusp height and enamel thickness shown here demonstrates the potential for variation in size and form of cusp relations, even after calcification has begun. This has been previously shown in crosssectional studies of hominid tooth germs of different stages of development and attributed to more rapid growth of the distal cusps (Butler, 1968; Siebert and Swindler, 1991). Modification of cuspal relationships following cusp initiation has also been demonstrated in developmental studies of vole and mouse tooth germs (Salazar-Ciudad and Jernvall, 2004). Our results demonstrate variation in growth and differentiation rates within the tooth throughout all stages of development. There is a mesio-distal gradient that is primarily associated with differing growth velocity and enamel matrix apposition and a bucco-lingual axis that affects cusp shape and height. The latter is expressed in differential growth and delayed coalescence between the metaconid and entoconid.

A recent study by Hlusco et al. (2004) on molar cusp patterning in pedigreed baboons concluded that there was some genetic independence between lophs on the same molar crown. Their findings are echoed in our demonstration of different levels of modality in cusp development within the human second deciduous molar and provide striking evidence of the potential for developmental variability. 

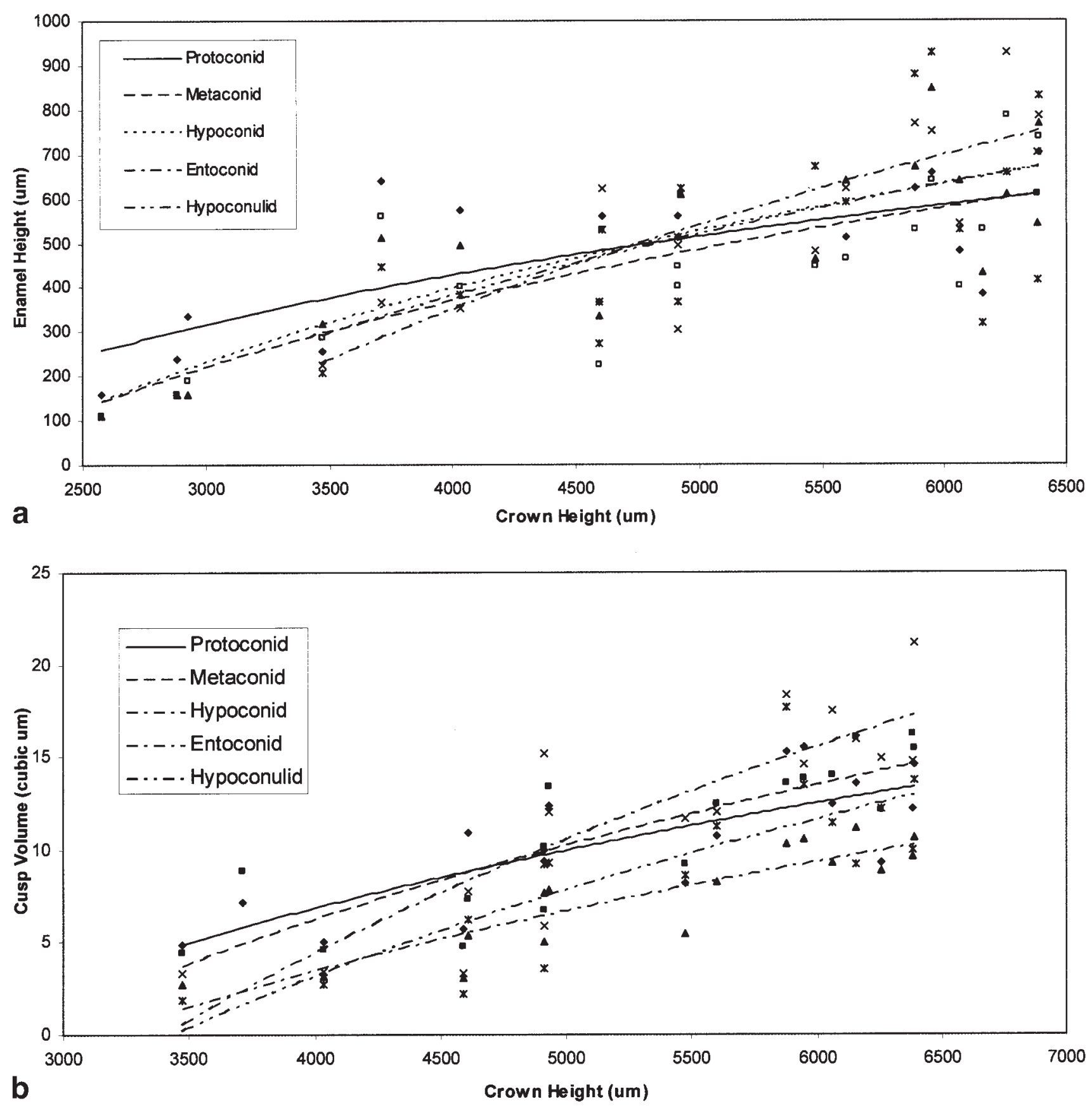

Fig. 3. Lines of best fit calculated for (a) enamel height and (b) enamel volume of each cusp at different stages of development. Both are positively correlated with crown height. But rank order of enamel volume in the completed tooth differs from rank order of enamel height over cusp tips.

\begin{tabular}{lcccr} 
& \multicolumn{2}{c}{ Enamel height } & \multicolumn{2}{c}{ Enamel volume } \\
\cline { 2 - 5 } Cusp & $\mathrm{R}^{2}$ & $P$ Value & $\mathrm{R}^{2}$ & 0.41 \\
Protoconid & 0.19 & 0.11 & 0.53 & 0.02 \\
Metaconid & 0.39 & 0.01 & 0.62 & 0.00 \\
Hypoconid & 0.57 & 0.02 & 0.72 & 0.00 \\
Entoconid & 0.51 & 0.01 & 0.76 & 0.00 \\
Hypoconulid & 0.51 & 0.01 & 0.00
\end{tabular}




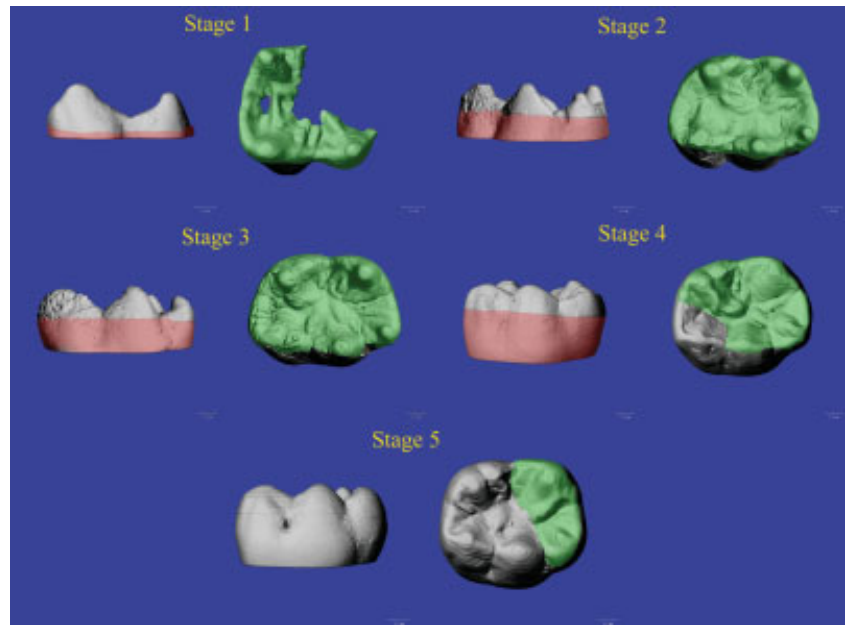

Fig. 4. Buccal and occlusal views of microCT reconstructions of lower left molars at different stages of development. Protoconid (Prd) is bottom left. Red coloring in buccal view indicates basal crown height, green coloring on occlusal view indicates developing ename matrix contributing to increase in enamel height. Note that enamel apposition on the protoconid (shown in gray on occlusal view) is complete by stage 4 , but still continuing on other cusps. [Color figure can be viewed in the online issue, which is available at www.interscience.wiley.com.]

McCollum and Sharpe (2001) pointed out that molar crown variation in fossil hominins could be apportioned into that associated with generalized enlargement of the entire crown versus that primarily affecting the later-developing talonid cusps. They proposed that these differences could be associated with the activity of growth factors regulating epithelial cell proliferation in initial bud formation or in the cap stage. Our study has focused on the deciduous molar in which growth is more rapid than that of the permanent molars. This means that the time lag between the cascade of cusp initiation and calcification is short. Our results demonstrate that the traditional demarcation into trigonid and talonid does not account for the pattern of development or extent of variation found. Rather, intraspecific variation in size of the second deciduous molar is expressed primarily in the metaconid, hypoconulid, and entoconid. These are the last cusps to coalesce with one another and their basal area reflects the continued expansion of the basal portion of the tooth (Figs. 1,2). This is why the order of coalescence, rather than order of initiation, constitutes the major source of variation in cusp area and fissure depth.

Our results also show that much of the variation in total crown height and volume occurs after cuspal union, and is related to basal crown height and enamel thickness. These results are not specific to the teeth examined here. Twin studies (Townsend et al., 2003) have shown that in permanent molars the later stages of crown development, expressed in maximum crown diameters, are less variable but more dimorphic than those of earlier stages represented by intercusp distances. They attributed this variation to the influence of epigenetic factors on the progressive infolding of the internal epithelium, but noted that the location of the cusp tips on the outer enamel surface of the tooth differs from that shown at the DEJ.

\section{CONCLUSIONS}

The model developed here for the lower second deciduous molar provides accurate definition and quantification of enamel and dentin. The partitioning of the tooth using the DEJ enables us to quantify growth in different locations within the tooth as represented by relative height of dentin cusp tips and their bases, basal crown height, and enamel thickness. Most important, the height of coalescence of cusps at the DEJ reflects order of coalescence and so provides a relative chronology of earlier versus later stages of dental ontogeny. We propose that this model will enable us to trace growth trajectories and variation in fossil teeth. Since the deciduous teeth complete most of their development before birth, the model presented here can also be applied to identify the onset of abnormal growth patterns in utero and their association with developmental abnormalities identified postnatally.

\section{REFERENCES}

Alexander JM, Bab I, Fish S, Muller R, Uchiyama T, Gronowicz G, Nahounou M, Zhao Q, White DW, Chorev M, Gazit D, Rosenblatt M. 2001. Human parathyroid hormone 1-34 reverses bone loss in ovariectomized mice. J Bone Miner Res 16:1665-1673.

Beynon AD. 1992. Circaseptan rhythms in enamel development in modern humans and Plio-Pleistocene hominids. In: Smith P, Tchernov E, editors. Structure function and evolution of teeth. Tel-Aviv: Freund. p 295-309.

Beynon AD, Dean MC. 1987. Crown-formation time of a fossil hominid premolar tooth. Arch Oral Biol 32:773-780.

Beynon AD, Wood BA. 1987. Patterns and rates of enamel growth in the molar teeth of early hominids. Nature 326:493-496.

Butler PM. 1968. Growth of the human second lower deciduous molar. Arch Oral Biol 13:671-682.

Butler PM. 1998. The relation of cusp development and calcification to growth. In: Mayhall J, Heikkinen T, editors. Dental morphology 1998: Proceedings of the 11th International Symposium on Dental Morphology. Oulu, Finland: Oulu University Press. p 26-32.

Conroy GC, Vannier MW. 1991. Dental development and the evolution of life history in Hominidae. Am J Phys Anthropol 86:137-156. Faerman M, Zilberman U, Smith P, Kharitonov V, Batsevitz V. 1994. A Neanderthal infant from the Barakai Cave, Western Caucasus. J Hum Evol 27:405-415.

Gabet Y, Muller R, Regev E, Sela J, Shteyer A, Salisbury K, Chorev M, Bab I. 2004. Osteogenic growth peptide modulates fracture callus structural and mechanical properties. Bone 35:65-73.

Gregory WK. 1922. The origin and evolution of the human dentition. Baltimore: Williams \& Wilkins.

Hlusko LJ, Maas ML, Mahaney MC. 2004. Statistical genetics of molar cusp patterning in pedigreed baboons: implications for primate dental development and evolution. J Exp Zool Part B Mol Dev Evol 302:268-283.

Jernvall J. 2000. Linking development with generation of novelty in mammalian teeth. Proc Natl Acad Sci U S A 97:2641-2645.

Jernvall J, Jung HS. 2000. Genotype, phenotype, and developmental biology of molar tooth characters. Am J Phys Anthropol Suppl 31:171-190.

Jernvall J, Aberg T, Kettunen P, Keranen S, Thesleff I. 1998. The life history of an embryonic signaling center: BMP-4 induces p21 and is associated with apoptosis in the mouse tooth enamel knot. Development 125:161-169.

Korenhof CA. 1979. The evolution of the lower molar pattern and remnants of the trigonid crests in man. Ned Tijdschr Tandheelkd $86: 6-31$.

Kraus BS. 1952. Morphological realtionships between enamel and dentin surfaces of lower first molar tooth. J Dent Res 31:248-256.

Kraus BS, Jordan RE. 1965. The human dentition before birth. Philadelphia: Lea \& Febiger.

Lumsden AG. 1988. Spatial organization of the epithelium and the role of neural crest cells in the initiation of the mammalian tooth germ. Development 103(Suppl):155-169.

Macho GA, Berner ME. 1993. Enamel thickness of human maxillary molars reconsidered. Am J Phys Anthropol 92:189-200.

McCollum M, Sharpe PT. 2001. Evolution and development of teeth. J Anat 199:153-159. 
Muller R, Hahn M, Vogel M, Delling G, Ruegsegger P. 1996. Morphometric analysis of noninvasively assessed bone biopsies: comparison of high-resolution computed tomography and histologic sections. Bone 18:215-220.

Muller R, Van Campenhout H, Van Damme B, Van Der Perre G, Dequeker J, Hildebrand T, Ruegsegger P. 1998. Morphometric analysis of human bone biopsies: a quantitative structural comparison of histological sections and micro-computed tomography. Bone 23:59-66.

Peters OA, Laib A, Ruegsegger P, Barbakow F. 2000. Three-dimensional analysis of root canal geometry by high-resolution computed tomography. J Dent Res 79:1405-1409.

Polly P. 1998. Variability, selection, and constraints: development and evolution in viverravid (Carnivora, Mammalia) molar morphology. Paleobiology 24:409-429.

Ruegsegger P, Koller B, Muller R. 1996. A microtomographic system for the nondestructive evaluation of bone architecture. Calcif Tissue Int 58:24-29.

Salazar-Ciudad I, Jernvall J. 2004. How different types of pattern formation mechanisms affect the evolution of form and development. Evol Dev 6:6-16.

Sasaki K, Kanazawa E. 1998. Morphological traits on the dentinoenamel junction of lower deciduous molar series. In: Mayhall $\mathrm{J}$, Heikkinen T, editors. Dental morphology 1998: Proceedings of the 11th International Symposium on Dental Morphology. Oulu, Finland: Oulu University Press. p 167-178.
Siebert J, Swindler DR. 1991. Perinatal dental development in the Chimpanzee (Pan troglodytes). Am J Phys Anthropol 86:287-295.

Smith P, Gomorri JM, Spitz S, Becker J. 1997. Model for the examination of evolutionary trends in tooth development. Am J Phys Anthropol 102:283-294.

Smith P, Gomori J, Shaked R, Haydenblit R, Joskowicz L. 1998. A computerized approach to reconstruction of growth patterns in hominid molar teeth. In: Mayhall J, Heikkinen T, editors. Dental morphology 1998: Proceedings of the 11th International Symposium on Dental Morphology. Oulu, Finland: Oulu University Press. p 388-397.

Smith TM, Martin LB, Leakey MG. 2003. Enamel thickness, microstructure and development in Afropithecus turkanensis. J Hum Evol 44:283-306.

Thesleff I. 2003. Epithelial-mesenchymal signalling regulating tooth morphogenesis. J Cell Sci 116:1647-1648.

Townsend G, Richards L, Hughes T. 2003. Molar intercuspal dimensions: genetic input to phenotypic variation. J Dent Res 82: $350-355$.

von Stechow D, Balto K, Stashenko P, Muller R. 2003. Three-dimensional quantitation of periradicular bone destruction by micro-computed tomography. J Endod 29:252-256.

Zilberman U, Smith P, Kupietzky A, Mass E. 2004. The effect of hereditary disorders on tooth components: a radiographic morphometric study of two syndromes. Arch Oral Biol 49:621-628. 